PROCEEDINGS OF THE

AMERICAN MATHEMATICAL SOCIETY

Volume 129, Number 10, Pages 2823-2831

S 0002-9939(01)05895-6

Article electronically published on February 15, 2001

\title{
A NUMERICAL CONDITION FOR A DEFORMATION OF A GORENSTEIN SURFACE SINGULARITY TO ADMIT A SIMULTANEOUS LOG-CANONICAL MODEL
}

\author{
TOMOHIRO OKUMA
}

(Communicated by Ron Donagi)

\begin{abstract}
Let $\pi: X \rightarrow T$ be a deformation of a normal Gorenstein surface singularity over the complex number field $\mathbb{C}$. We assume that $T$ is a neighborhood of the origin of $\mathbb{C}$. Then we prove that $\pi$ admits a simultaneous log-canonical model if and only if an invariant $-P_{t} \cdot P_{t}$ of each fiber $X_{t}$ is constant.
\end{abstract}

\section{INTRODUCTION}

Let $\pi: X \rightarrow T$ be a deformation of a normal Gorenstein surface singularity over the complex number field $\mathbb{C}$. We assume that $T$ is a sufficiently small neighborhood of the origin of $\mathbb{C}$. In his paper [8], Laufer proved that the deformation admits a simultaneous canonical model if and only if $-K_{t} \cdot K_{t}$ is constant. In this paper, we prove the log-version of Laufer's results. Instead of $-K \cdot K$, we adopt an invariant $-P \cdot P$. It is a numerical invariant of a normal surface singularity, and its fundamental properties are stated in [14]. For example, the equality below is proved in [14, Introduction]:

$$
-P \cdot P / 2=\limsup _{m \rightarrow \infty} \delta_{m} / m^{2} .
$$

In this equality, $\delta_{m}$ denotes the $m$-th $L^{2}$-plurigenus of the singularity. Since $\delta_{m}\left(X_{t}\right)$ is upper semicontinuous for any $m$ by [3], it follows from the equality (1.1) that $-P \cdot P$ is upper semicontinuous. Our main theorem is the following:

Theorem 1.1. The following conditions are equivalent:

(1) $\pi$ admits a simultaneous log-canonical model;

(2) $-P_{t} \cdot P_{t}$ is constant.

The implication $(1) \Rightarrow(2)$ follows from the invariance of the log-plurigenus $\lambda_{m}\left(X_{t}\right)$ with $m>>0$ and an equality similar to (1.1). Let $f: Y \rightarrow X$ be a log-canonical model of $X$ with the exceptional divisor $E$. Then $f_{t}: Y_{t} \rightarrow X_{t}$ is a $\log$-canonical model for $t \in T \backslash\{0\}$ near 0 (Lemma 3.1). We define sheaves $\mathcal{I}_{m}$ and $\mathcal{A}_{m}$ by $\mathcal{I}_{m}=f_{*} \mathcal{O}_{Y}\left(m\left(K_{Y}+E\right)\right)$ and $\mathcal{A}_{m}=\psi_{*} \mathcal{O}_{M}\left(m\left(K_{M}+A\right)\right)$, where

Received by the editors August 10, 1998 and, in revised form, July 15, 1999, November 4, 1999, and February 7, 2000.

2000 Mathematics Subject Classification. Primary 14B07; Secondary 14E15, 32S30, 32S45.

Key words and phrases. Normal Gorenstein surface singularity, plurigenera, log-canonical model. 
$\psi:(M, A) \rightarrow\left(X_{0}, x\right)$ is a good resolution. Since $Y_{0}=\operatorname{Proj} \bigoplus_{m \geq 0} \mathcal{I}_{m} \otimes \mathbb{C}(0)$, the implication $(2) \Rightarrow(1)$ follows from the claim that if $-P_{t} \cdot P_{t}$ is constant, then $\mathcal{I}_{m} \otimes \mathbb{C}(0)=\mathcal{A}_{m}$. To show the claim, we will use Izumi's results on the analytic orders [6] (precisely, Ishii's version [4]) and formulas for the plurigenera, and prove the torsion freeness of the sheaf $\mathcal{O}_{X}\left(m K_{X}\right) / \mathcal{I}_{m}$ (Lemma 4.8). We will also prove that if a morphism $f: Y \rightarrow X$ is a simultaneous log-canonical model, then it is a log-canonical model of $X$ (Lemma 4.2). Our methods will be used in higher dimensions (Remark 4.12).

We denote by $\mathbb{N}, \mathbb{Q}$ and $\mathbb{R}$ the set of positive integers, the set of rational numbers and the set of real numbers, respectively.

Thanks are due to Professor J. Wahl for precious suggestions. Thanks are also due to the referee for reading the paper carefully and giving helpful suggestions.

\section{Preliminaries}

2.1. Log-canonical models. Let $X$ be a normal variety over $\mathbb{C}$ of dimension $d \geq 2$. Let $D=\sum d_{i} D_{i}$ be a $\mathbb{Q}$-divisor on $X$, where the $D_{i}$ are distinct prime divisors and $d_{i} \in \mathbb{Q}$. We put $D_{\text {red }}=\sum_{d_{i} \neq 0} D_{i}$. Let $f: Y \rightarrow X$ be a birational morphism of normal varieties and $E$ the maximal reduced exceptional divisor on $Y$. For a divisor $D$ on $X$, we denote by $f_{*}^{-1}(D)$ the strict transform of $D$ under the morphism $f$. The morphism $f: Y \rightarrow X$ is called a good resolution of a pair $(X, D)$, if $Y$ is nonsingular and $\left(f_{*}^{-1}(D)+E\right)_{\text {red }}$ is a divisor with only simple normal crossings. A $\mathbb{Q}$-divisor $B$ on $X$ is called a boundary if it satisfies $0 \leq B \leq B_{\text {red }}$.

Definition 2.1. Let $B$ be a boundary on $X$. A divisor $K_{X}+B$ is said to be log-canonical if the following conditions are satisfied:

(1) $K_{X}+B$ is a $\mathbb{Q}$-Cartier divisor.

(2) There exists a good resolution $f: Y \rightarrow X$ of $(X, B)$ such that

$$
K_{Y}+f_{*}^{-1}(B)=f^{*}\left(K_{X}+B\right)+\sum a_{i} E_{i}
$$

for $a_{i} \in \mathbb{Q}$ with the condition that $a_{i} \geq-1$, where the $E_{i}$ vary all the exceptional prime divisors on $Y$.

If $K_{X}+B$ is log-canonical, then the equality in condition (2) above is satisfied with $a_{i} \geq-1$ for any resolution of $X$ (see [7, Lemma 0-2-12]). Hence we have the following:

Lemma 2.2. Let $f: Y \rightarrow X$ be any good resolution of a pair $(X, B)$ with the exceptional divisor $E$, and let $B_{Y}=f_{*}^{-1}(B)+E$. If $K_{X}+B$ is log-canonical, then we have $f_{*} \mathcal{O}_{Y}\left(m\left(K_{Y}+B_{Y}\right)\right)=\mathcal{O}_{X}\left(m\left(K_{X}+B\right)\right)$ for any $m \in \mathbb{N}$.

Definition 2.3. Let $f: Y \rightarrow X$ be a birational morphism with the exceptional divisor $E$. Then the morphism $f: Y \rightarrow X$ is called a $\log$-canonical model of $X$, if the divisor $K_{Y}+E$ is log-canonical and $f$-ample.

Theorem 2.4 (see [1, 6.16]). Let $X$ be a normal variety of dimension $d \leq 3$. Then there exists a unique log-canonical model $f: Y \rightarrow X$ of $X$. In fact, the following morphism is the log-canonical model:

$$
\operatorname{Proj} \bigoplus_{n \geq 0} h_{*} \mathcal{O}_{Z}\left(n\left(K_{Z}+F\right)\right) \rightarrow X
$$

where $h: Z \rightarrow X$ is any proper birational morphism with the exceptional divisor $F$ such that the divisor $K_{Z}+F$ is log-canonical. 
2.2. Plurigenera. Let $(X, x)$ be a normal isolated singularity and $f:(M, A) \rightarrow$ $(X, x)$ a good resolution of the singularity $(X, x)$, where $A$ is the exceptional divisor.

Definition 2.5 (cf. 9], [15]). We define the log-plurigenera $\left\{\lambda_{m}(X, x)\right\}_{m \in \mathbb{N}}$ and the $L^{2}$-plurigenera $\left\{\delta_{m}(X, x)\right\}_{m \in \mathbb{N}}$ by

$$
\begin{aligned}
\lambda_{m}(X, x) & =\operatorname{dim}_{\mathbb{C}} \mathcal{O}_{X}\left(m K_{X}\right) / f_{*} \mathcal{O}_{M}\left(m\left(K_{M}+A\right)\right), \\
\delta_{m}(X, x) & =\operatorname{dim}_{\mathbb{C}} \mathcal{O}_{X}\left(m K_{X}\right) / f_{*} \mathcal{O}_{M}\left(m\left(K_{M}+A\right)-A\right) .
\end{aligned}
$$

These definitions are independent of the choice of a good resolution.

Proposition 2.6. Let $g: Y \rightarrow X$ be a partial resolution with the exceptional divisor $E$ such that $K_{Y}+E$ is log-canonical. Then we have

$$
\lambda_{m}(X, x)=\operatorname{dim}_{\mathbb{C}} \mathcal{O}_{X}\left(m K_{X}\right) / g_{*} \mathcal{O}_{Y}\left(m\left(K_{Y}+E\right)\right) .
$$

Proof. It follows from Lemma 2.2.

Let $(X, x)$ be a normal surface singularity and $f:(M, A) \rightarrow(X, x)$ the minimal good resolution. Let $K$ be a canonical divisor on $M$. Let $A=\bigcup_{i=1}^{k} A_{i}$ be the decomposition of $A$ into irreducible components. By [12] Theorem A.1], $K+A$ admits a unique Zariski decomposition $P+N$, where $P$ and $N$ are elements of $\sum_{i=1}^{k} \mathbb{Q} A_{i}$ satisfying the following:

(1) $(K+A) \cdot A_{i}=(P+N) \cdot A_{i}$ for all $i$;

(2) $P$ is $f$-nef and $N$ is effective;

(3) $P \cdot N=0$.

Theorem 2.7 (see 14], 11]). For every $m \in \mathbb{N}$, we have

$$
\begin{aligned}
& \lambda_{m}(X, x)=-(P \cdot P) m^{2} / 2+(P \cdot K) m / 2+b_{1}(m), \\
& \delta_{m}(X, x)=-(P \cdot P)(m-1)^{2} / 2-(P \cdot K)(m-1) / 2+b_{2}(m),
\end{aligned}
$$

where $b_{1}(m)$ and $b_{2}(m)$ are bounded functions of $m$.

\section{Deformations and some invariants}

Let $\pi: X \rightarrow T$ be a deformation of a normal Gorenstein surface singularity $\left(X_{0}, x\right)=\pi^{-1}(0)$, where $T$ is a neighborhood of the origin of $\mathbb{C}$. Then $X$ is a Gorenstein variety. Therefore, for any $t \in T$, we have $\mathcal{O}_{X_{t}}\left(m K_{X}\right) \cong \mathcal{O}_{X_{t}}\left(m K_{X_{t}}\right)$. For any morphism $h: W \rightarrow X$, we denote by $W_{t}$ the fiber $(\pi \circ h)^{-1}(t)$ and set $h_{t}=\left.h\right|_{W_{t}}$. Let $f: Y \rightarrow X$ be the log-canonical model of $X$ with the maximal reduced exceptional divisor $E$ on $Y$. Then $\mathcal{O}_{Y}\left(m\left(K_{Y}+E\right)\right)$ is a Cohen-Macaulay $\mathcal{O}_{Y}$-module for $m \in \mathbb{N}$. We define sheaves $\mathcal{I}_{m}$ and $\mathcal{Q}_{m}$ by $\mathcal{I}_{m}=f_{*} \mathcal{O}_{Y}\left(m\left(K_{Y}+E\right)\right)$ and $\mathcal{Q}_{m}=\mathcal{O}_{X}\left(m K_{X}\right) / \mathcal{I}_{m}$ for every $m \in \mathbb{N}$. Put $T^{*}=T \backslash\{0\}$. We assume that $T$ is small enough to satisfy the following conditions:

(1) there exists a good resolution $g: M \rightarrow Y$ of $(Y, E)$ which induces a good resolution $M_{t} \rightarrow X_{t}$ for any $t \in T^{*}$;

(2) $Y_{t}$ is a normal surface with the exceptional divisor $E_{t}=E \cap Y_{t}$ for any $t \in T^{*}$.

Lemma 3.1. For any $t \in T^{*}$, the restriction $f_{t}: Y_{t} \rightarrow X_{t}$ is the log-canonical model of $X_{t}$. 
Proof. Let $t$ be a point of $T^{*}$. Since the normal sheaf of $Y_{t}$ in $Y$ is trivial and $\mathcal{O}_{Y}\left(m\left(K_{Y}+E\right)\right)$ is Cohen-Macaulay, we obtain that

$$
\mathcal{O}_{Y_{t}}\left(m\left(K_{Y}+E\right)\right) \cong \mathcal{O}_{Y_{t}}\left(m\left(K_{Y_{t}}+E_{t}\right)\right)
$$

for every $m \in \mathbb{N}$. Hence the $f_{t}$-ampleness of the divisor $K_{Y_{t}}+E_{t}$ follows from the $f$-ampleness of $K_{Y}+E$. Let $g: M \rightarrow Y$ be a resolution as in (1) above. Let $F$ be the exceptional divisor of $g$. Then we obtain that

$$
K_{M}+g_{*}^{-1}(E)+F=g^{*}\left(K_{Y}+E\right)+\Delta,
$$

where $\Delta$ is an effective divisor supported in $F$. Restricting those divisors to $M_{t}$, we see that $K_{Y_{t}}+E_{t}$ is $\log$-canonical.

Let $W$ be a normal variety whose singular locus $W_{\text {sing }}$ is a finite set. Then we put

$$
\lambda_{m}(W)=\sum_{w \in W_{\text {sing }}} \lambda_{m}(W, w) \text { and } \delta_{m}(W)=\sum_{w \in W_{\text {sing }}} \delta_{m}(W, w) .
$$

Let $\psi(t): M_{t} \rightarrow X_{t}$ be the minimal good resolution of the singularities and $K_{t}$ the canonical divisor on $M_{t}$. Let $A_{t, p}$ be a connected component of the exceptional set $A_{t}$ on $M_{t}$ which blows down to $p \in\left(X_{t}\right)_{\text {sing }}$. Let $P_{t, p}+N_{t, p}$ be the Zariski decomposition of $K_{t}+A_{t, p}$, where $P_{t, p}$ and $N_{t, p}$ are $\mathbb{Q}$-divisors supported in $A_{t, p}$. We define a $\mathbb{Q}$-divisor $P_{t}$ on $M_{t}$ by $P_{t}=\sum_{p \in\left(X_{t}\right)_{s i n g}} P_{t, p}$, and regard $-P_{t} \cdot P_{t}$ as a function of $t$.

Proposition 3.2. For any $m \in \mathbb{N}$, we obtain that

$$
\begin{aligned}
& \lambda_{m}\left(X_{t}\right)=-\left(P_{t} \cdot P_{t}\right) m^{2} / 2+\left(P_{t} \cdot K_{t}\right) m / 2+b_{t}(m), \\
& \delta_{m}\left(X_{t}\right)=-\left(P_{t} \cdot P_{t}\right)(m-1)^{2} / 2-\left(P_{t} \cdot K_{t}\right)(m-1) / 2+b_{t}^{\prime}(m),
\end{aligned}
$$

where $b_{t}$ and $b_{t}^{\prime}$ are bounded functions of $m$. Furthermore, $-P_{t} \cdot P_{t}$ is upper semicontinuous.

Proof. It follows from Theorem 2.7 and Introduction.

Let $\mathbb{C}(t)$ be the residue field of $t \in T$, i.e., $\mathbb{C}(t)=\mathcal{O}_{T, t} / \mathcal{M}_{t}$, where $\mathcal{M}_{t}$ is the maximal ideal. We write $\otimes \mathbb{C}(t)$ instead of $\otimes_{\mathcal{O}_{T}} \mathbb{C}(t)$. By Nakayama's lemma, we obtain the inequality

$$
\operatorname{dim}_{\mathbb{C}} \mathcal{Q}_{m} \otimes \mathbb{C}(t) \leq \operatorname{dim}_{\mathbb{C}} \mathcal{Q}_{m} \otimes \mathbb{C}(0) .
$$

Let $\mathcal{I}_{m, 0}$ be the image of the natural homomorphism $\mathcal{I}_{m} \otimes \mathbb{C}(0) \rightarrow \mathcal{O}_{X_{0}}\left(m K_{X_{0}}\right)$. Then $\mathcal{Q}_{m} \otimes \mathbb{C}(0) \cong \mathcal{O}_{X_{0}}\left(m K_{X_{0}}\right) / \mathcal{I}_{m, 0}$.

Lemma 3.3. The following conditions are equivalent:

(1) $\operatorname{dim}_{\mathbb{C}} \mathcal{Q}_{m} \otimes \mathbb{C}(t)=\operatorname{dim}_{\mathbb{C}} \mathcal{Q}_{m} \otimes \mathbb{C}(0)$ for $t \in T^{*}$;

(2) $\mathcal{Q}_{m}$ is a torsion free $\mathcal{O}_{T}$-module;

(3) $\mathcal{I}_{m} \otimes \mathbb{C}(0)=\mathcal{I}_{m, 0}$.

Lemma 3.4. There exists a closed analytic subset $S \subset T$ such that $\lambda_{m}\left(X_{t}\right)=$ $\operatorname{dim}_{\mathbb{C}} \mathcal{Q}_{m} \otimes \mathbb{C}(t)$ for any $t \in T \backslash S$ and any $m \in \mathbb{N}$.

Proof. There exists an integer $m_{0}$ such that $R^{1} f_{*} \mathcal{O}_{Y}\left(m\left(K_{Y}+E\right)\right)=0$ for $m>$ $m_{0}$. Let $S$ be a closed analytic subset of $T$ such that the $\mathcal{O}_{T}$-coherent sheaf 
$R^{1} f_{*} \mathcal{O}_{Y}\left(m\left(K_{Y}+E\right)\right)$ is torsion free outside $S$ for $m \leq m_{0}$. Then, for any $t \in T \backslash S$, we have the exact sequence

$$
\begin{aligned}
& 0 \rightarrow f_{*} \mathcal{O}_{Y}\left(m\left(K_{Y}+E\right)\right) \stackrel{\times \tau}{\longrightarrow} f_{*} \mathcal{O}_{Y}\left(m\left(K_{Y}+E\right)\right) \rightarrow f_{*} \mathcal{O}_{Y_{t}}\left(m\left(K_{Y_{t}}+E_{t}\right)\right) \\
& \rightarrow R^{1} f_{*} \mathcal{O}_{Y}\left(m\left(K_{Y}+E\right)\right) \stackrel{\times \tau}{\longrightarrow} R^{1} f_{*} \mathcal{O}_{Y}\left(m\left(K_{Y}+E\right)\right),
\end{aligned}
$$

where $\tau$ is a local parameter at $t$. Since the last arrow is injective, it follows that $f_{*} \mathcal{O}_{Y_{t}}\left(m\left(K_{Y_{t}}+E_{t}\right)\right)$ is the image of $f_{*} \mathcal{O}_{Y}\left(m\left(K_{Y}+E\right)\right)$. Hence we obtain that

$$
\mathcal{Q}_{m} \otimes \mathbb{C}(t)=\mathcal{O}_{X_{t}}\left(m K_{X_{t}}\right) / f_{t *} \mathcal{O}_{Y_{t}}\left(m\left(K_{Y_{t}}+E_{t}\right)\right) .
$$

Now the assertion follows from Proposition 2.6 and Lemma 3.1

Let $\psi:(M, A) \rightarrow\left(X_{0}, x\right)$ be a good resolution. For each $m \in \mathbb{N}$, we put $\mathcal{A}_{m}=$ $\psi_{*} \mathcal{O}_{M}\left(m\left(K_{M}+A\right)\right)$ and define invariants $\epsilon_{m}$ and $\theta_{m}$ by

$$
\begin{aligned}
\epsilon_{m} & =\operatorname{dim}_{\mathbb{C}} \mathcal{A}_{m} /\left(\mathcal{I}_{m, 0} \cap \mathcal{A}_{m}\right), \quad \text { and } \\
\theta_{m} & =\operatorname{dim}_{\mathbb{C}} \mathcal{I}_{m, 0} /\left(\mathcal{A}_{m} \cap \mathcal{I}_{m, 0}\right), \quad \text { respectively. }
\end{aligned}
$$

Using Proposition 3.4 and the equality (3.1), we obtain the inequality

$$
\lambda_{m}\left(X_{t}\right) \leq \lambda_{m}\left(X_{0}\right)+\epsilon_{m}-\theta_{m} .
$$

Lemma 3.5. There exist $a, b \in \mathbb{Q}$ such that $\epsilon_{m} \leq a m+b$.

Proof. Let $\omega$ be a section of $\psi_{*} \mathcal{O}_{M}\left(m\left(K_{M}+A\right)-A\right)$. By [3, Theorem 2.1], there exists a section $\omega^{\prime}$ of $f_{*} \mathcal{O}_{Y}\left(m\left(K_{Y}+E\right)-E\right)$ such that the image of $\omega^{\prime}$ in $\mathcal{O}_{X_{0}}\left(m K_{X_{0}}\right)$ is $\omega$. Since $f_{*} \mathcal{O}_{Y}\left(m\left(K_{Y}+E\right)-E\right) \subset \mathcal{I}_{m}$, we see that $\omega$ belongs to $\mathcal{I}_{m, 0}$. Hence we obtain that $\psi_{*} \mathcal{O}_{M}\left(m K_{M}+(m-1) A\right) \subset \mathcal{I}_{m, 0} \cap \mathcal{A}_{m}$. This implies that

$$
\epsilon_{m} \leq \operatorname{dim}_{\mathbb{C}} \mathcal{A}_{m} / \psi_{*} \mathcal{O}_{M}\left(m K_{M}+(m-1) A\right)=\delta_{m}\left(X_{0}, x\right)-\lambda_{m}\left(X_{0}, x\right) .
$$

From Theorem 2.7, we obtain the assertion.

\section{THE MAIN THEOREM}

In this section, we prove the main theorem. Let $\pi: X \rightarrow T$ be as in the preceding section.

Definition 4.1. Let $f: Y \rightarrow X$ be a birational morphism with the maximal reduced exceptional divisor $E$. Suppose that $K_{Y}+E$ is $\mathbb{Q}$-Cartier. We call $f$ a simultaneous log-canonical model if for any $t \in T, f_{t}: Y_{t} \rightarrow X_{t}$ is the log-canonical model of $X_{t}$ and $E_{t}$ is a reduced divisor.

A simultaneous log-canonical model of $\pi: X \rightarrow T$ is unique if it exists. In fact, we have the following:

Lemma 4.2. Let $f: Y \rightarrow X$ be a simultaneous log-canonical model with the exceptional divisor $E$. Then $f$ is the log-canonical model of $X$ and $E_{t}$ is the exceptional set on $Y_{t}$ for any $t \in T$.

Proof. Let $B$ be the union of the curves $C$ on $Y$ such that $C \nsubseteq E$ and $f(C)=\{x\}$, the singular point of $X_{0}$. Then $E \cup B$ is the exceptional set of $f$, and hence $E_{0} \cup B$ is the exceptional set of $f_{0}$. Since $X$ is Gorenstein, we may assume that $\left(K_{Y_{0}}\right)_{\text {red }} \subset E_{0}$. By assumption, $K_{Y_{0}}+E_{0}+B$ is $f_{0}$-ample. Thus there exists a positive divisor $D$ supported on $E_{0} \cup B$ such that $K_{Y_{0}}+E_{0}+B=-D$. Hence $B$ is void, and $E_{0}$ is the exceptional set of $f_{0}$. Since $K_{Y_{t}}+E_{t}$ is $f_{t}$-ample for any $t \in T$, we see that $K_{Y}+E$ is $f$-ample. By inversion of adjunction [1, Chap. 17], $K_{Y}+E$ is log-canonical, since $K_{Y_{t}}+E_{t}$ is log-canonical for any $t \in T$. 
Remark 4.3. In the situation above, we see that $h^{0}\left(\mathcal{O}_{E_{t}}\right)=1$ for any $t$. This implies that $E_{t}$ is connected. Hence $X_{t}$ has at most one non-log-canonical singularity.

In the following, the notation is the same as in the preceding section, unless otherwise specified; so $f: Y \rightarrow X$ denotes the canonical model of $X$. We assume that $T$ is sufficiently small in each case. For each $m \in \mathbb{N}$, we regard $\lambda_{m}\left(X_{t}\right)$ as a function of $t$.

Proposition 4.4. Suppose that $f: Y \rightarrow X$ is the simultaneous log-canonical model of the deformation $\pi: X \rightarrow T$. Then $\lambda_{m}\left(X_{t}\right)$ is constant for $m>>0$.

Proof. Consider the exact sequence

$$
\begin{aligned}
0 \rightarrow f_{*} \mathcal{O}_{Y}\left(m\left(K_{Y}+E\right)\right) & \stackrel{\times \tau}{\longrightarrow} f_{*} \mathcal{O}_{Y}\left(m\left(K_{Y}+E\right)\right) \rightarrow f_{*} \mathcal{O}_{Y_{0}}\left(m\left(K_{Y_{0}}+E_{0}\right)\right) \\
& \rightarrow R^{1} f_{*} \mathcal{O}_{Y}\left(m\left(K_{Y}+E\right)\right),
\end{aligned}
$$

where $\tau$ is a local parameter at 0 . Since $K_{Y}+E$ is $f$-ample, $R^{1} f_{*} \mathcal{O}_{Y}\left(m\left(K_{Y}+E\right)\right)=$ 0 for $m>>0$. We fix such an integer $m$. Then $f_{*} \mathcal{O}_{Y_{0}}\left(m\left(K_{Y_{0}}+E_{0}\right)\right)=\mathcal{I}_{m} \otimes \mathbb{C}(0)$. It is clear that $\mathcal{I}_{m} \otimes \mathbb{C}(0)=\mathcal{I}_{m, 0}$. Thus $\operatorname{dim}_{\mathbb{C}} \mathcal{Q}_{m} \otimes \mathbb{C}(0)=\lambda_{m}\left(X_{0}\right)$ by Proposition 2.6 Then Lemma 3.3 and 3.4 imply that $\lambda_{m}\left(X_{t}\right)=\lambda_{m}\left(X_{0}\right)$ for $t \in T^{*}$.

In [13, Tomari and Watanabe proved a result on the order of growth of the $L^{2}$-plurigenera by applying Izumi's results on the analytic orders [6]. We will use their argument. The following lemma is Ishii's version [4, Lemma 1.5].

Lemma 4.5. Let $(W, w)$ be a d-dimensional normal isolated singularity, and let $h: W_{1} \rightarrow W$ be a resolution of the singularity which factors through the blowing up by the maximal ideal of the singular point. Let $F=\bigcup_{i=1}^{k} F_{i}$ be the exceptional divisor on $W_{1}$, where the $F_{i}$ are irreducible components. Then there exist positive numbers $\beta \in \mathbb{R}$ and $b \in \mathbb{N}$ such that:

For an $\mathcal{O}_{W}$-ideal $J=h_{*} \mathcal{O}_{W_{1}}\left(-\sum_{i=1}^{k} a_{i} F_{i}\right)$ with $a_{i}>b$ for some $i$, the inequalities $\operatorname{dim}_{\mathbb{C}} \mathcal{O}_{W} / J \geq \beta\left(a_{i}\right)^{d}(i=1, \ldots, k)$ hold.

Lemma 4.6. Assume that $-P_{t} \cdot P_{t}$ is constant. Then $\mathcal{I}_{m, 0} \subset \mathcal{A}_{m}$ for all $m \in \mathbb{N}$.

Proof. It suffices to show that $\theta_{m}=0$ for all $m \in \mathbb{N}$. Note that Proposition 3.2 the inequality (3.2) and Lemma 3.5 imply that $\theta_{m}$ is bounded by a linear function. Assume that $\theta_{r} \neq 0$. Let $\omega$ be a section of $\mathcal{I}_{r, 0}$ which does not belong to $\mathcal{A}_{r}$. We define a homomorphism $\varphi_{m}: \mathcal{O}_{X_{0}} \rightarrow \mathcal{I}_{m r, 0}$ by $\varphi_{m}(s)=s \omega^{m}$ for every $m \in \mathbb{N}$. We denote by $J_{m}$ the inverse image $\varphi_{m}^{-1}\left(\mathcal{A}_{m r} \cap \mathcal{I}_{m r, 0}\right)$. Then we have the injection

$$
\mathcal{O}_{X_{0}} / J_{m} \rightarrow \mathcal{I}_{m r, 0} /\left(\mathcal{A}_{m r} \cap \mathcal{I}_{m r, 0}\right) \text {. }
$$

We put $a_{i}=\min \left\{v_{i}(\omega)+r, 0\right\}$, where $v_{i}$ is the valuation at an irreducible component $A_{i}$ of $A$. Then $J_{m}=\psi_{*} \mathcal{O}_{M}\left(\sum m a_{i} A_{i}\right)$. By the choice of $\omega$, there exists a component $A_{i}$ such that $a_{i}<0$. We may assume that the good resolution $\psi:(M, A) \rightarrow\left(X_{0}, x\right)$ factors through the blowing up by the maximal ideal of the point $x$. By Lemma 4.5, there exists a positive number $c \in \mathbb{R}$ such that $\theta_{m r} \geq \mathrm{cm}^{2}$ for any $m \in \mathbb{N}$. It contradicts the fact noted above.

The technique above is used in the proof of [5, Theorem 1]. In [5], a claim similar to " $\mathcal{A}_{m} \subset \mathcal{I}_{m, 0}$ " is proved by using [10, Lemma 1]. The upper semi-continuity of the plurigenus $\gamma_{m}$ follows from the claim. Unfortunately we have no log-version of [10, Lemma 1]. However, we can show the claim " $\mathcal{A}_{m} \subset \mathcal{I}_{m, 0}$ " under the condition that $-P_{t} \cdot P_{t}$ is constant. 
Lemma 4.7. Let $F$ be any prime divisor on $Y$ such that $f(F)=\{x\}$. Then there exists $m>0$ such that $f_{*} \mathcal{O}_{Y}\left(m\left(K_{Y}+E\right)+F\right) / \mathcal{I}_{m} \neq 0$.

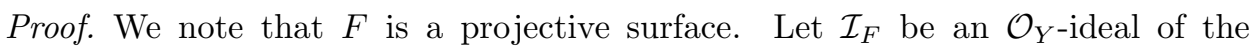
subvariety $F$, and let $L_{m}=m\left(K_{Y}+E\right)$. Since $L_{1}$ is $f$-ample, there exists an integer $n \in \mathbb{N}$ such that $\mathcal{O}_{F}\left(L_{n}\right)$ is a very ample invertible sheaf and the following sequence is exact for any $m \in \mathbb{N}$ :

$$
0 \rightarrow f_{*}\left(\mathcal{I}_{F} \mathcal{O}_{Y}\left(L_{m n}+F\right)\right) \rightarrow f_{*} \mathcal{O}_{Y}\left(L_{m n}+F\right) \rightarrow H^{0}\left(\mathcal{O}_{F}\left(L_{m n}+F\right)\right) \rightarrow 0 .
$$

By [2, III, Ex. 5.2], there exists a polynomial $q^{\prime}$ of degree 2 such that

$$
\operatorname{dim}_{\mathbb{C}} f_{*} \mathcal{O}_{Y}\left(L_{m n}+F\right) / f_{*}\left(\mathcal{I}_{F} \mathcal{O}_{Y}\left(L_{m n}+F\right)\right)=q^{\prime}(m)
$$

for $m>>$. Since $\mathcal{I}_{F} \mathcal{O}_{Y}\left(L_{m n}+F\right)$ is isomorphic to $\mathcal{O}_{Y}\left(L_{m n}\right)$ outside a onedimensional subvariety of $F$, there exists a polynomial $q$ of degree 2 such that $\operatorname{dim}_{\mathbb{C}} f_{*} \mathcal{O}_{Y}\left(L_{m n}+F\right) / \mathcal{I}_{m n} \geq q(m)$ for $m>>0$.

Lemma 4.8. Assume that $-P_{t} \cdot P_{t}$ is constant. Then $\mathcal{Q}_{m}$ is a torsion free $\mathcal{O}_{T}{ }^{-}$ module for any $m \in \mathbb{N}$.

Proof. Assume that $\mathcal{Q}_{m}$ has a torsion element. Then there exists the maximal reduced divisor $D \neq 0$ on $Y$ such that $f(D)=\{x\}$. Let $h: Z \rightarrow Y$ be the blowing up by an ideal sheaf $\mathcal{O}_{Y}(-D)$. Then $h$ is an isomorphism in codimension one and $h_{*}^{-1}(D)$ is a Cartier divisor. Thus there exists a prime divisor $F \subset h_{*}^{-1}(D)$ such that the intersection $F \cap(f \circ h)_{*}^{-1}\left(X_{0}\right)$ is one-dimensional. By Lemma 4.7 there exist $r>0$ and $\omega \in \mathcal{O}_{X}\left(r K_{X}\right)$ with the condition that the image of $\omega$ in $\mathcal{Q}_{r}$ is a torsion element and $v_{F}(\omega)<-r$, where $v_{F}$ denotes the valuation at $F$. Suppose that $v_{F}(\omega) \leq v_{F}\left(\omega^{\prime}\right)$ for all $\omega^{\prime} \in \mathcal{O}_{X}\left(r K_{X}\right)$ with the condition above. We denote by $\eta_{1}\left(\right.$ resp. $\left.\eta_{2}\right)$ the image of $\eta \in \mathcal{O}_{X}\left(m K_{X}\right)$ in $\mathcal{Q}_{m} \otimes \mathbb{C}(0)\left(\right.$ resp. $\left.\mathcal{O}_{X_{0}}\left(m K_{X_{0}}\right) / \mathcal{A}_{m}\right)$. We may assume that the good resolution $\psi:(M, A) \rightarrow\left(X_{0}, x\right)$ factors through $(f \circ h)_{*}^{-1}\left(X_{0}\right) \rightarrow X_{0}$. We denote by $\mathcal{T}_{m}$ the torsion submodule of $\mathcal{Q}_{m}$ for $m \in \mathbb{N}$. By the choice of $\omega, \omega_{1} \in \mathcal{T}_{r} \otimes \mathbb{C}(0)$, and $\omega_{1}$ and $\omega_{2}$ are nonzero. For each $m \in \mathbb{N}$, define a homomorphism $\varphi_{m}: \mathcal{O}_{X_{0}} \rightarrow \mathcal{O}_{X_{0}}\left(m r K_{X_{0}}\right) / \mathcal{A}_{m r}$ by $\varphi_{m}(s)=\left(s \omega^{m}\right)_{2}$. By an argument similar to that in the proof of Lemma 4.6. there exists a positive number $c$ such that $\operatorname{dim}_{\mathbb{C}} \varphi_{m}\left(\mathcal{O}_{X_{0}}\right) \geq \mathrm{cm}^{2}$. By Lemma 4.6, we have the natural surjection $\mathcal{Q}_{m r} \otimes \mathbb{C}(0) \rightarrow \mathcal{O}_{X_{0}}\left(m r K_{X_{0}}\right) / \mathcal{A}_{m r}$. Since the image of $\mathcal{T}_{m r} \otimes \mathbb{C}(0)$ in $\mathcal{O}_{X_{0}}\left(m r K_{X_{0}}\right) / \mathcal{A}_{m r}$ contains $\varphi_{m}\left(\mathcal{O}_{X_{0}}\right)$, we have $\operatorname{dim}_{\mathbb{C}} \mathcal{T}_{m r} \otimes \mathbb{C}(0) \geq \mathrm{cm}^{2}$. Then, for $t \in T^{*}$, we have the inequality

$$
\operatorname{dim}_{\mathbb{C}} \mathcal{Q}_{m r} \otimes \mathbb{C}(t) \leq \operatorname{dim}_{\mathbb{C}} \mathcal{Q}_{m r} \otimes \mathbb{C}(0)-c m^{2} .
$$

This implies the following:

$$
\lambda_{m r}\left(X_{t}\right) \leq \lambda_{m r}\left(X_{0}\right)+\varepsilon_{m r}-c m^{2} .
$$

By Proposition 3.2 and Lemma 3.5 we get $-P_{t} \cdot P_{t}<-P_{0} \cdot P_{0}$.

Remark 4.9. Assume that $-P_{t} \cdot P_{t}$ is constant. From the proof above, we see that $Y_{0}$ is irreducible. Hence any irreducible component of $E$ dominates $T$. Since $Y_{0}$ is a principal divisor, for any irreducible component $F$ of $E$, the intersection $F \cap Y_{0}$ is a one-dimensional variety.

Lemma 4.10. Assume that $-P_{t} \cdot P_{t}$ is constant. Then $\mathcal{I}_{m, 0}=\mathcal{A}_{m}$ for any $m \in \mathbb{N}$. 
Proof. By Lemma 4.6 it suffices to show that $\mathcal{A}_{m} \subset \mathcal{I}_{m, 0}$. Let $\omega$ be a section of $\mathcal{A}_{m}$ and $\omega^{\prime}$ a section of $\mathcal{O}_{X}\left(m K_{X}\right)$ whose image in $\mathcal{O}_{X_{0}}\left(m K_{X_{0}}\right)$ is $\omega$. We may assume that $\psi:(M, A) \rightarrow\left(X_{0}, x\right)$ factors through $f_{0}: Y_{0} \rightarrow X_{0}$. If $v_{F}\left(\omega^{\prime}\right)<-m$ for an irreducible component $F$ of $E$, then there exists an irreducible component $A_{i}$ of $A$ lying over the variety $F \cap Y_{0}$ such that $v_{A_{i}}\left(\psi^{*} \omega\right)<-m$. It contradicts the choice of $\omega$. Hence $\omega^{\prime}$ belongs to $\mathcal{I}_{m}$, and $\omega$ belongs to $\mathcal{I}_{m, 0}$.

Now we are ready to prove the main theorem which is the log-version of Laufer's result on simultaneous canonical models.

Theorem 4.11. The following conditions are equivalent:

(1) $\pi: X \rightarrow T$ admits the simultaneous log-canonical model.

(2) The function $\lambda_{m}\left(X_{t}\right)$ of $t$ is constant for any $m \in \mathbb{N}$.

(3) The function $-P_{t} \cdot P_{t}$ of $t$ is constant.

Proof. Consider the condition: $(2)^{\prime} \lambda_{m}\left(X_{t}\right)$ is constant for $m>>0$. By Proposition 3.2 and 4.4 we obtain the implication $(1) \Rightarrow(2)^{\prime} \Rightarrow(3)$. We assume that $-P_{t} \cdot P_{t}$ is constant. Then, from Lemma 3.34 .8 and 4.10 we obtain the following equalities for any $m \in \mathbb{N}$ :

$$
\mathcal{I}_{m} \otimes \mathbb{C}(0)=\mathcal{I}_{m, 0}=\mathcal{A}_{m}, \quad \operatorname{dim}_{\mathbb{C}} \mathcal{Q}_{m} \otimes \mathbb{C}(t)=\operatorname{dim}_{\mathbb{C}} \mathcal{Q}_{m} \otimes \mathbb{C}(0) .
$$

Now it is clear that (2) holds, and that $Y_{0}=\operatorname{Proj}\left(\bigoplus_{m>0} \mathcal{I}_{m} \otimes \mathbb{C}(0)\right)$ is the logcanonical model of $X_{0}$. Since $\mathcal{A}_{m}=f_{*} \mathcal{O}_{Y_{0}}\left(m\left(K_{Y_{0}}+E_{0}\right)\right.$ ) for $m>>$ (cf. the proof of Proposition 4.4), and $K_{Y_{0}}+E_{0}$ is $f_{0}$-ample, we see that $E_{0}$ is reduced. On the other hand, $f_{t}: Y_{t} \rightarrow X_{t}$ is the log-canonical model for $t \in T^{*}$ by Lemma 3.1 Hence we obtain the condition (1).

Remark 4.12. The techniques in this paper can be used in higher dimensions. In fact, we can generalize the main theorem to the higher-dimensional case if the following claims hold:

(1) there exists a log-canonical model $f: Y \rightarrow X$ of $X$;

(2) for an isolated singularity $(W, w)$ of dimension $n$, there exists $p=p(W, w) \in$ $\mathbb{R}$ such that $\lambda_{m}(W, w)=p m^{n}+O\left(m^{n-1}\right)$.

Note that $\delta_{m}(W, w)=\lambda_{m}(W, w)+O\left(m^{n-1}\right)$ by an argument in [14, p. 627].

\section{REFERENCES}

[1] J. Kollár et al, Flips and Abundance for Algebraic Threefolds, Astérisque, vol. 211, Soc. Math. France, 1992. MR 94f:14013

[2] R. Hartshorne, Algebraic Geometry, Graduate Texts in Mathematics, vol. 52, Springer-Verlag, New York, Heidelberg, Berlin, 1977. MR 57:3116

[3] S. Ishii, Small deformation of normal singularities, Math. Ann. 275 (1986), 139-148. MR 87i: 14003

[4] - The asymptotic behavior of plurigenera for a normal isolated singularity, Math. Ann. 286 (1990), 803-812. MR 91d:32049

[5] Simultaneous canonical models of deformations of isolated singularities, Algebraic Geometry and Analytic Geometry (A. Fujiki et.al., ed.), ICM-90 Satell. Conf. Proc., SpringerVerlag, 1991, pp. 81-100. MR 94j:14002

[6] S. Izumi, A measure of integrity for local analytic algebras, Publ. Res. Inst. Math. Sci. 21 (1985), 719-735. MR 87i:32014

[7] Y. Kawamata, K. Matsuda, and K. Matsuki, Introduction to the minimal model problem, Algebraic geometry, Sendai 1985 (T. Oda, ed.), Advanced Studies in Pure Math., vol. 10, Kinokuniya, Tokyo, North-Holland, Amsterdam, 1987, pp. 283-360. MR 89e:14015 
[8] H. Laufer, Weak simultaneous resolution for deformations of Gorenstein surface singularities, Singularities (P. Orlik, ed.), Proc. Sympos. Pure Math., vol. 40, Part 2, Amer. Math. Soc., 1983, pp. 1-30. MR 84k:32030

[9] M. Morales, Resolution of quasi-homogeneous singularities and plurigenera, Compositio Math. 64 (1987), 311-327. MR 88j:14004

[10] N. Nakayama, Invariance of the plurigenera of algebraic varieties under minimal model conjecture, Topology 25, No. 2 (1986), 237-251. MR 87g:14034

[11] T. Okuma, The plurigenera of Gorenstein surface singularities, Manuscripta Math. 94 (1997), 187-194. MR 98k:14050

[12] F. Sakai, Anticanonical models of rational surfaces, Math. Ann. 269 (1984), 389-410. MR 85m:14058

[13] M. Tomari and K. Watanabe, On $L^{2}$-plurigenera of not-log-canonical Gorenstein isolated singularities, Proc. Amer. Math. Soc. 109 (1990), 931-935. MR 91m:32043

[14] J. Wahl, A characteristic number for links of surface singularities, J. Amer. Math. Soc. 3 (1990), 625-637. MR 91c:14043

[15] K. Watanabe, On plurigenera of normal isolated singularities, I, Math. Ann. 250 (1980), 65-94. MR 82f:32025

Department of Mathematics, Gunma National College of Technology, 580 Toriba, Maebashi, Gunma 371, JaPAN

E-mail address: okuma@nat.gunma-ct.ac.jp 